\title{
Effets d'une ration riche en protéines et pauvre en cellulose sur les performances de croissance, le développement des organes et la composition de la carcasse chez le lapin Blanc de Termonde
}

\author{
R. POISMANS, P.J. WITTOUCK \\ Université Catholique de Louvain \\ Laboratoire de Physiologie des Animaux domestiques \\ B.1348 Louvain-la-Neuve, Belgique
}

\begin{abstract}
Résumé
La présente étude a permis de mettre en évidence la manière dont le lapin s'adapte à une ration déséquilibrée et dans quelle mesure les composants de celle-ci sont utilisés par l'animal.

Deux groupes de 18 lapins, des deux sexes et de la race Blanc de Termonde, ont disposé ad libitum, bien avant le sevrage et jusqu'à l'âge de 120 jours, d'un régime expérimental ou d'un régime de référence. Le régime de référence (aliment I) contenait 18,9 p. 100 de protéines et 20,2 p. 100 de cellulose. Le régime expérimental (aliment II), fort déséquilibré, contenait un excès de protéines $(27,0 \mathrm{p}, 100)$ et était légèrement carencé en fibres $(12,4$ p. 100). Les mesures ont porté sur les performances de croissance, le développement de certains organes, la composition de la carcasse, ainsi que des graisses de dépôts.

L'absence de maladie, de même que de bonnes performances de croissance montrent que les lapins s'adaptent bien aux deux rations. L'aliment II, riche en protéines, permet aux lapins d'atteindre un poids moyen plus élevé ( $3491 \mathrm{~g})$ que ceux qui ont reçu l'aliment I (2965 g). Dans le cas du groupe II, l'indice de consommation $(3,22)$ est cependant plus faible que celui de l'autre groupe $(3,78)$. Les CUDa de la MS et de la MAT de l'aliment II sont meilleurs que ceux de l'aliment $I$; néanmoins, les lapins qui ont reçu ce dernier fixent mieux l'azote qu'ils reçoivent.

Les graisses de dépôts sont plus développées chez les animaux du groupe II (aliment expérimental). Le rendement de la carcasse est plus faible chez ces derniers (61,8 p. 100) que chez ceux qui reçoivent l'aliment I $(65,1$ p. 100). Le poids moyen des carcasses des animaux du groupe II est plus élevé et les muscles plus riches en matière sèche et matières grasses. Par contre, le taux de MAT du muscle des animaux recevant l'aliment pauvre en protéines (groupe I) est plus important.
\end{abstract}

Dans aucun cas, le sexe des animaux n'a montré une influence sur les paramètres étudiés, et cela quel que soit l'aliment distribué.

Mots clés : lapins, ration riche en protéines et pauvre en cellulose, performances de croissance, composition de la carcasse. 


\section{Introduction}

Parmi les recherches concernant la nutrition du lapin, rares sont celles qui portent sur les effets dus à un déséquilibre simultané des deux importants nutriments que sont la cellulose et les protéines. La plupart des études se sont intéressées à un manque ou un excès d'un des nutriments de la ration, les autres étant maintenus à un niveau moyen. Des recherches ont permis de déterminer qu'un aliment fournissant de 2300 à $2800 \mathrm{kcal}$ d'ED $/ \mathrm{kg}$ est suffisant pour couvrir les besoins énergétiques du lapin, celui-ci étant d'ailleurs capable d'ajuster son ingestion alimentaire en fonction de ses besoins en énergie (Lebas, 1973 a ; Dehalle, 1980 ; Reyne \& Salcedo-Miliani, 1981).

Chez le lapin, les meilleurs résultats sont obtenus avec des taux alimentaires de 15 à 18 p. 100 de protéines (Lebas, 1973 a ; Colin, 1974 ; LebenHartz, 1974), et de 12 à 16 p. 100 de cellulose (Lebas, 1979 ; Soyeux-Salavize, 1975 ; Spreadbury, 1978).

Dans la présente étude, nous avons tenté d'établir la possibilité d'une adaptation précoce du lapin à un régime simultanément hypocellulosique $(12,4$ p. 100) et hyperprotéinique $(27,0 \mathrm{p}$. 100). La concentration énergétique de l'aliment préparé était élevée, soit $2783 \mathrm{kcal} / \mathrm{kg}$. Les proportions de l'aliment de référence étaient de 20,2 p. 100 de cellulose et de 18,9 p. 100 de protéines, avec une concentration énergétique de $2414 \mathrm{kcal} / \mathrm{kg}$. Le rapport protéines digestibles/énergie digestible (PD/ED) était de $67 \mathrm{~g} / 1000 \mathrm{kcal}$ ED pour l'aliment testé et de $49 \mathrm{~g}$ pour l'aliment de référence.

L'analyse a porté sur les performances de croissance, le développement de certains organes du tube digestif et la composition de la carcasse.

\section{I1. Matériel et méthodes}

\section{A. Les animaux}

L'expérimentation a porté sur 36 lapins de la race Blanc de Termonde, issus de 6 génitrices, âgées de 6 mois et primipares. Au sein des nichées, les lapereaux choisis se rapprochaient d'un poids moyen de $470 \mathrm{~g}$ au sevrage. Ils furent divisés en deux groupes, contenant autant de mâles que de femelles. Bien avant le sevrage ( $30 \mathrm{j}$.$) ,$ les lapereaux recevaient déjà l'aliment, qui leur serait donné tout au long de l'expérience. Les animaux ont été élevés ensuite en cage individuelle, jusqu'à l'âge de 4 mois.

La période d'observation s'est donc étendue de 31 à 120 jours. Durant cette période, les animaux ont été nourris ad libitum et ont disposé d'eau à volonté. Le rapport éclairement/obscurité était maintenu à $12 \mathrm{~h} / 12 \mathrm{~h}$; tandis que la température était de $20^{\circ} \mathrm{C} \pm 1$ et l'humidité relative comprise entre 70 et 80 p. 100.

Les quantités d'aliment ingéré et d'eau consommée ont été mesurées quotidiennement et individuellement. Les animaux ont été pesés hebdomadairement, durant la matinée. Les deux dernières pesées ont été faites au début et à la fin du jeûne de 24 heures auquel ils étaient soumis avant l'abattage. Les 36 animaux ont été sacrifiés à âge constant, soit 12 semaines. 
Parallèlement 4 lapins, 2 mâles et 2 femelles, de la même race, âgés de 10 semaines, furent placés en cage à métabolisme, pour mesurer l'utilisation digestive des différents composants des deux rations. Pour tester les différents facteurs, nous avons organisé notre plan expérimental en «cross over». Les animaux ont été pesés au début et à la fin de chaque période. Les quantités d'aliment ingéré, l'eau consommée, les urines et les fèces émises ont été mesurées quotidiennement. Les CUD $_{\mathrm{a}}$ des aliments et de leurs éléments nutritifs furent étudiés, après des collectes de 5 jours, et ont été calculés par les méthodes classiques.

\section{B. Les aliments}

Les aliments distribués aux animaux, sous forme de granulés, ont été fabriqués en notre laboratoire. La composition de ces aliments, ainsi que leurs caractéristiques physiques et chimiques sont données dans le tableau 1. La paille de blé a été substituée au tourteau de soja dans le cas de l'aliment de référence. Les teneurs en protéines diffèrent suivant l'apport respectif de tourteau de soja.

\section{TABLEAU 1}

Composition centésimale des régimes expérimentaux, analyse chimique et caractéristiques des granulés.

Composition of experimental diets expressed as percentage, chemical analysis and pellets characteristics.

\begin{tabular}{|c|c|c|c|}
\hline & $\begin{array}{c}\text { Aliment } \mathrm{I} \\
\text { Diet I }\end{array}$ & Aliment II & \\
\hline \multicolumn{3}{|l|}{ Composants $(\%)$} & Components $(\%)$ \\
\hline Orge $\ldots$ & 39,4 & 39,4 & Barley \\
\hline Luzerne & 19,7 & 19,7 & Lucerne \\
\hline Soja ....... & 19,7 & 39,4 & Soya \\
\hline Paille de blé ..... & 19,7 & 0 & Wheat straw \\
\hline Min. + Vit. $11 \ldots$ & 1,5 & 1,5 & Minerals and vitamins (1) \\
\hline \multicolumn{2}{|l|}{ Composition chimique } & \multicolumn{2}{|r|}{ Chemical composition } \\
\hline Matière sèche $(\%)$ & 88,3 & 88,4 & Dry matter (\%) \\
\hline Cendres $(\% \quad$ MS) $\ldots \ldots \ldots$ & 6,7 & 6,9 & Ash $(\% \quad D M)$ \\
\hline Matières azotées ( $\%$ MS) & 18,9 & 27,0 & Crude protein (\% DM) \\
\hline Energie digestible $(\mathrm{kcal} / \mathrm{kg})$ & 2.414 & 2.783 & Digestible energy $(\mathrm{kcal} / \mathrm{kg})$ \\
\hline Protéines digestibles $(\mathrm{g} / 100 \mathrm{~g}) \ldots$ & 11,6 & 18,3 & Digestible protein $(\mathrm{g} / \mathrm{l} / 00 \mathrm{~g})$ \\
\hline $\mathrm{g}$ protéines digestibles $/ 1000 \mathrm{kcal}$ & & & $g$ digestible protein/1000 $\mathrm{kcal}$ \\
\hline ED $\ldots \ldots \ldots \ldots \ldots \ldots \ldots$ & 48,5 & 67,3 & $D E$ \\
\hline Cellulose brute $(\% \quad$ MS) $\ldots$. & 20,2 & 12,4 & Crude fibre (\% DM) \\
\hline Matière grasse $(\%$ MS $) \ldots \ldots \ldots$ & 3,9 & 4,6 & Fat $(\% \quad D M)$ \\
\hline Caractéristiques des granulés (2) & \multicolumn{3}{|r|}{ Pellets characteristics (ㄹ) } \\
\hline Diamètre (mm) & 4,8 & 4,8 & Diameter $(\mathrm{mm})$ \\
\hline Longueur (mm) & 11,0 & 9,9 & Length $(\mathrm{mm})$ \\
\hline Dureté $(\mathrm{kg} \mathrm{Kahl}) \ldots \ldots \ldots \ldots$ & 29,0 & 20,0 & Hardness (kg Kahl) \\
\hline
\end{tabular}

(1) Protector S.A. : «Aliment C $80 »-« C 80$ diet».

(2) Moyenne obtenue à partir de 50 mesures - Mean value of 50 measurements. 
Les concentrations en énergie digestible des deux aliments furent obtenues à partir des teneurs et des CUD des nutriments suivant la formule de JENTSCH et al. (1963).

\section{Les paramètres mesurés après l'abattage}

Plusieurs manipulations ont été effectuées :

a. Du sang a été prélevé chez chaque animal, au niveau de la veine cave, afin d'obtenir un échantillon de sérum pour le dosage de l'urée et des protéines sériques.

b. Les graisses de dépôt au niveau du cœur, des reins et des organes génitaux ont été pesées. $1 \mathrm{~g}$ de graisse périrénale fut prélevée sur chaque animal, afin de déterminer, après fixation au tétraoxyde d'osmium, le nombre et la taille des adipocytes. Les dosages et les calibrages furent réalisés à l'aide d'un Coulter Counter.

c. La carcasse chaude, mais vide, avec la tête, les pattes et les manchons fut pesée. Le rendement à l'abattage fut calculé en rapportant le poids de la carcasse chaude au poids dư lapin après un jeûne de 24 heures (poids carcasse/poids à jeûn).

d. De la carcasse, plusieurs muscles ont été prélevés. Sur la cuisse gauche, les muscles coccygéofemoralis et biceps fémoris; le triceps gauche. Ces muscles ont été pesés, numérotés puis congelés. Leurs teneurs en matière sèche (MS), matière azotée totale (MAT) et matière grasse (MG) ont été déterminées ensuite. Les MAT et MG ont été obtenues respectivement par la méthode KJELDAHL et par extraction à froid au $\mathrm{CCl}_{4}$.

e. Une portion du tendeur au fascia lata et une portion du muscle rectus abdominus ont été immergées dans du Bouin Allen pour être conservées. Après traitement, des coupes semi-fines $(6 \mu)$ de ces muscles ont été fixées sur lame, afin d'apprécier microscopiquement l'importance des dépôts graisseux intra-musculaires.

f. Le fémur et l'humérus gauche ont été détachés et nettoyés pour en déterminer le poids et la taille. Ils furent mesurés suivant le modèle proposé par Olivier (1960).

g. Les principaux organes (cœur, poumons, foie, rate) ont été pesés séparément. Les viscères vides (estomac, intestin grêle, caecum et appendice, côlons proximal et distal) ont été pesés et mesurés.

\section{Analyses statistiques}

Toutes les analyses statistiques furent réalisées sur ordinateur APPLE II. Les séries de données ont été étudiées par analyse de variance, soit par un modèle de deux critères croisés fixes (aliments et sexes), soit par un modèle à un critère fixe -4 répétitions (aliments).

\section{Résultats}

\section{A. Performances de croissance}

Après 90 jours d'expérimentation, les animaux du groupe II (aliment riche en protéines) ont atteint le poids moyen de $3491 \mathrm{~g}$ et consommé $9753 \mathrm{~g}$ de l'aliment II ; tandis que ceux du groupe $\mathrm{I}$, avec une consommation totale de $9478 \mathrm{~g}$ de l'aliment I, n'ont atteint que $2965 \mathrm{~g}$ de poids moyen (tabl. 2). 
TABleau 2

Performances moyennes d'engraissement \pm Erreur Standard. Mean fattening performance \pm Standard Error.

\begin{tabular}{|c|c|c|}
\hline & $\begin{array}{l}\text { Aliment I } \\
\text { Diet I }\end{array}$ & $\begin{array}{l}\text { Aliment II } \\
\text { Diet II }\end{array}$ \\
\hline $\begin{array}{l}\text { Poids vif initial }(30 \mathrm{j})(\mathrm{g}) \ldots \ldots \ldots \ldots \ldots \ldots \ldots \\
\text { Initial live weight }(30 \mathrm{~d})(\mathrm{g})\end{array}$ & $464 \pm 20$ & $471 \pm 13$ \\
\hline $\begin{array}{l}\text { Poids vif final }(120 \mathrm{j})(\mathrm{g}) \ldots \ldots \ldots \ldots \ldots \ldots \ldots \\
\text { Final live weight }(120 \text { d) }(g)\end{array}$ & $2965 \pm 53$ & $3491 \pm 85$ \\
\hline $\begin{array}{l}\text { Gain moyen quotidien }(g / j) \ldots \ldots \ldots \ldots \ldots \ldots \\
\text { Daily mean gain }(g / d)\end{array}$ & 29 & 35 \\
\hline $\begin{array}{l}\text { Consommation totale }(g) \ldots \ldots \ldots \ldots \ldots \ldots \ldots \\
\text { Total feed intake }(g)\end{array}$ & $9478 \pm 202$ & $9753 \pm 267$ \\
\hline $\begin{array}{l}\text { Consommation quotidienne }(\mathrm{g}) \\
\text { Daily feed intake }(\mathrm{g})\end{array}$ & 105 & 108 \\
\hline $\begin{array}{l}\text { Indice de consommation } \ldots \ldots \ldots \ldots \ldots \ldots \ldots \ldots \\
\text { Feed conversion ratio }\end{array}$ & 3,78 & 3,22 \\
\hline
\end{tabular}

L'évolution des poids, considérée semaine par semaine, et représentée graphiquement (fig. 1), montre qu'il n'y a pas de différence significative $(P>0,05)$ entre les poids moyens des lapins des deux groupes jusqu'à la $7^{\circ}$ semaine après le sevrage. Au cours de la $8^{\prime \prime}$ semaine, la différence devient hautement significative $(P<0,01)$ et elle ne cessera de s'accentuer jusqu'à la fin de l'expérience.

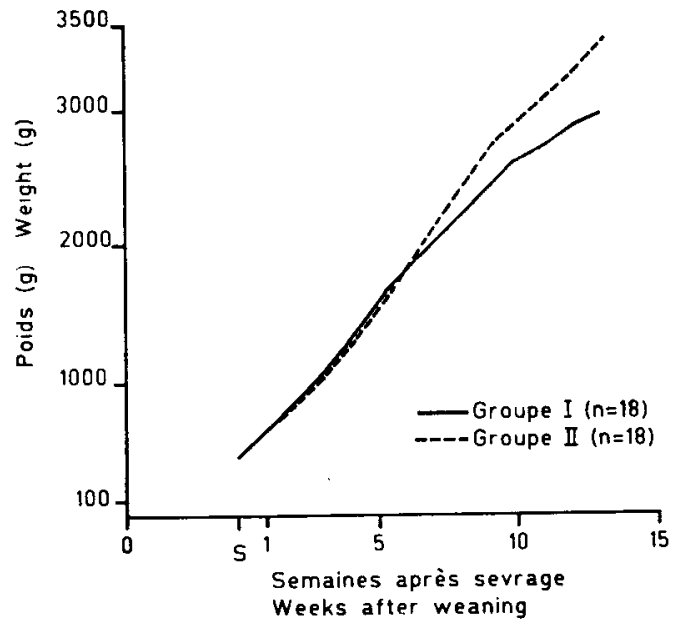

FIG. 1

Evolution des performances hebdomadaires moyennes de croissance pondérale.

Variation in weekly weight gain. 
Au moment de l'abattage, la courbe de croissance des animaux alimentés avec l'aliment I (pauvre en protéines et riche en cellulose) commence à fléchir, alors que la vitesse de croissance des autres animaux reste constante (fig. 1). Pour chacune des courbes, nous avons établi une droite de régression (fig. 2).

L'absence d'impact des différences de poids au sevrage a été confirmée en rapportant tous les poids à une base commune, soit 467,5 g, le poids moyen de 36 lapins au début de l'expérience. De même l'analyse statistique d'un éventuel effet dû au sexe, au sein des groupes, n'a pas montré de différences significatives $(P>0,05)$.

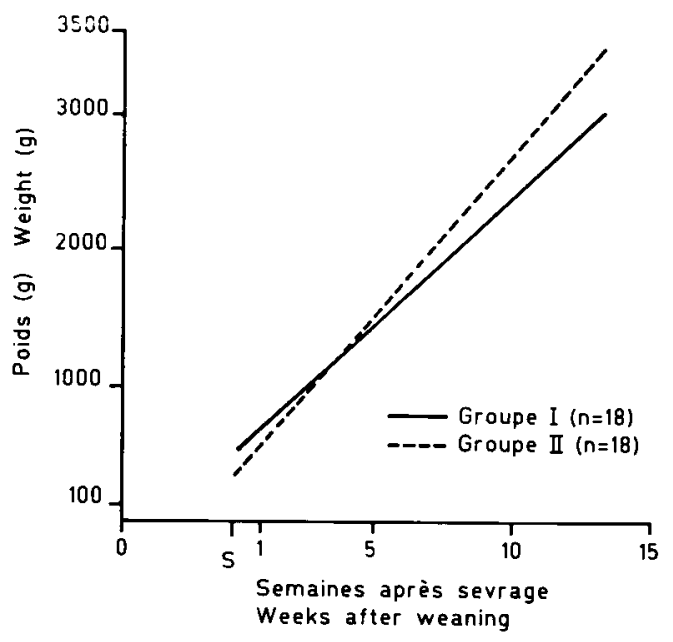

Fig. 2

Droites de régression.

Regression lines.

\section{B. Etude des consommations}

La comparaison des quantités totales consommées par les lapins des deux groupes ne montre pas de différence importante (tabl. 2). La distribution dans le temps des consommations alimentaires (en kcal E.D.) de chaque groupe doit cependant être précisée (fig. 3). En effet, il apparaît que la période de croissance est divisée en deux temps : jusqu'à la $6^{\circ}$ semaine après le sevrage, l'ingestion est plus importante chez les animaux du groupe I ; ensuite, ce sont les animaux du groupe II qui consomment le plus.

Etant donné que chez le lapin, la quantité ingérée dépend surtout de l'âge physiologique, déterminé en grande partie par le poids plutôt que par l'âge réel, les quantités ingérées (en kcal E.D.) ont également été exprimées en fonction du poids métabolique (fig. 4). L'orientation observée est fort similaire à celle des consommations exprimées en fonction du temps. Les droites de régression correspondant aux courbes de la figure 4 montrent, qu'à poids métabolique égal, les animaux du groupe II consomment plus que ceux du groupe I. Cette différence s'accentue au fur et à mesure que les animaux deviennent plus lourds. 


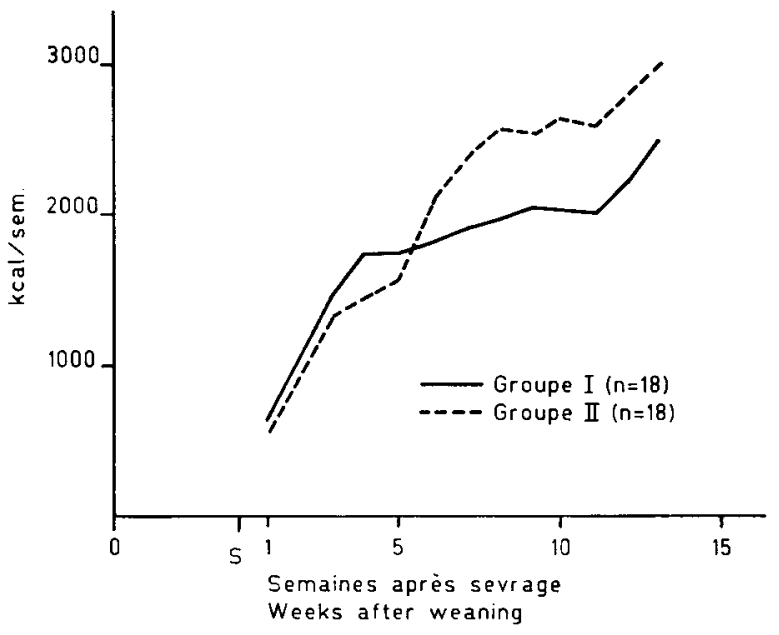

FIG. 3

Energie digestible ingérée par semaine entre 1 et 13 semaines.

Weekly digestible energy intake between 1 and 13 weeks.

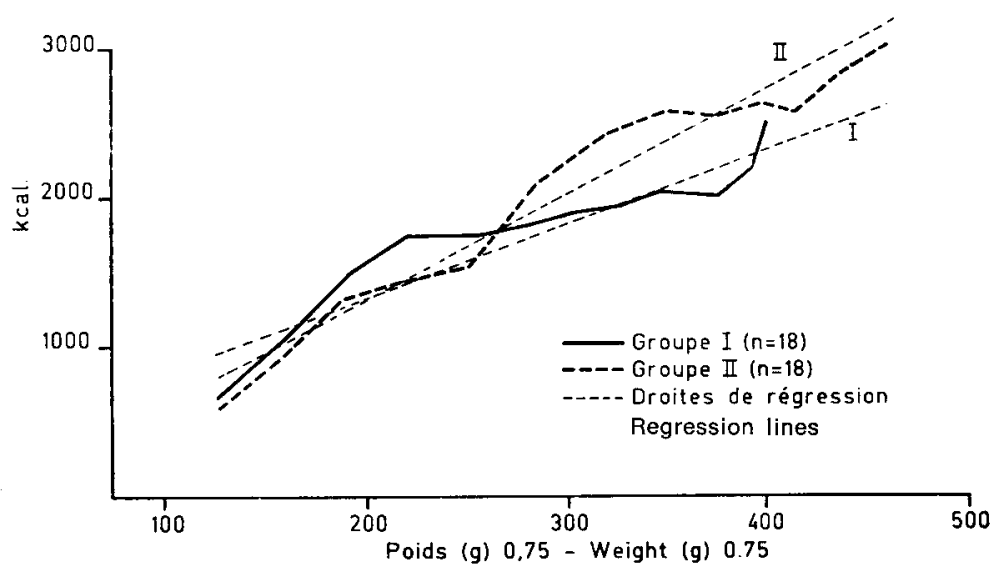

FIG. 4

Energie digestible ingérée en fonction du poids métabolique.

Digestibility energy intake as a function of metabolic weight.

Par ailleurs, à consommation pratiquement égale au cours de la seconde phase de la croissance, les animaux du groupe II réalisent une croissance bien supérieure à ceux du groupe I. L'indice de consommation (IC) fut de 3,22 pour le groupe II et de 3,78 pour le groupe I. L'évolution des IC en fonction du temps est fort semblable pour les deux groupes, 1'I.C. des lapins nourris avec l'aliment II, riche en 
protéines, étant cependant toujours inférieur à celui des animaux qui ont reçu l'aliment I. Les animaux du groupe II augmentent en moyenne leur IC de 0,38 unité par semaine et ceux du groupe I de 0,45 unité par semaine (fig. 5).

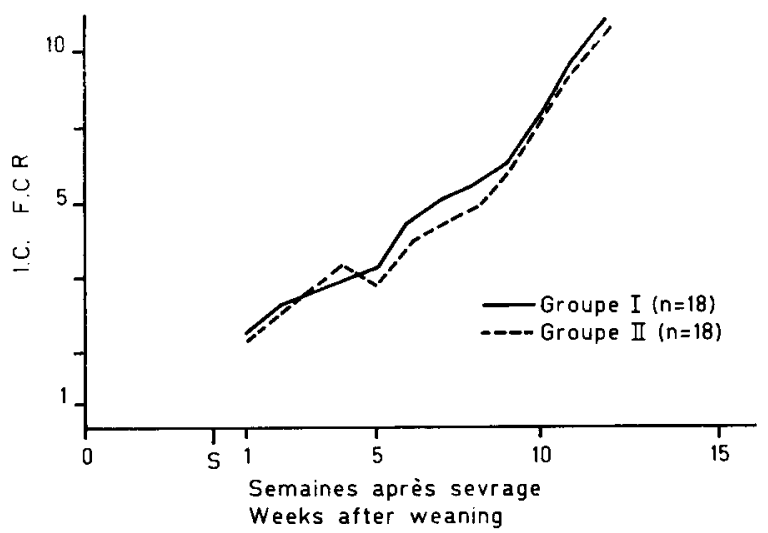

FIG. 5

Evolution de l'indice de consommation (ingéré kcal/gain de poids g) des lapins entre 1 et 12 semaines d'âge.

Variation in feed conversion ratio of rabbits between 1 and 12 weeks of age.

\section{TABLEAU 3}

Digestibilités apparentes (\%) ( \pm Ecart-type). Apparent digestibilities $(\%)( \pm$ Standard Deviation).

\begin{tabular}{l|c|c|c}
\hline \multicolumn{1}{c|}{ CUDa \% } & $\begin{array}{c}\text { Aliment I } \\
\text { Diet I }\end{array}$ & $\begin{array}{c}\text { Aliment II } \\
\text { Diet II }\end{array}$ & $\begin{array}{c}\text { Niveau } \\
\text { de signification } \\
\text { Significance }\end{array}$ \\
\hline $\begin{array}{l}\text { Matière sèche } \ldots \ldots \ldots \ldots \ldots \\
\text { Dry matter }\end{array}$ & $60,1 \pm 2,9$ & $67,5 \pm 2,9$ & $\mathbf{P}<0,05$ \\
$\begin{array}{l}\text { Matières azotées totales .... } \\
\text { Crude protein }\end{array}$ & $69,3 \pm 2,5$ \\
$\begin{array}{l}\text { Cellulose brute .......... } \\
\text { Crude fibre }\end{array}$ & $22,7 \pm 2,4$ \\
$\begin{array}{l}\text { Lipides ................. } \\
\text { Fat }\end{array}$ & $97,4 \pm 0,8$ & $\mathbf{7 6 , 6 \pm 3 , 4}$ & $\mathbf{P}<0,05$ \\
\hline
\end{tabular}

\section{Digestibilité des aliments}

Les digestibilités apparentes de la matière sèche (MS) ot de la matière azotée totale (MAT), estimées par le calcul du coefficient d'utilisation digestive apparente (CUDa), diminuent dans la même proportion sous l'effet de la cellulose. 
Les CUDa de la cellulose brute et de la matière grasse n'ont montré aucune différence significative $(P>0,05)$ en fonction de l'aliment (tabl. 3 et 4 ).

\section{Mesures effectuées après l'abattage}

Les performances zootechniques observées dans les deux groupes de lapins (tabl. 5) ne furent pas équivalentes. Le poids moyen de carcasse est significativement plus élevé $(P<0,05)$ chez les animaux du groupe II (aliment riche en protéines), que chez ceux du groupe I, alors que ces derniers présentent un meilleur rendement de carcasse $(P<0,01)$.

\section{TABLEAU 4}

Bilan azoté $(X \pm D . S$.$) .$

Nitrogen balance $(X \pm S . D$.).

\begin{tabular}{|c|c|c|c|}
\hline & $\begin{array}{c}\text { Aliment } \mathrm{I} \\
\text { Diet }\end{array}$ & $\begin{array}{l}\text { Aliment II } \\
\text { Diet II }\end{array}$ & $\begin{array}{c}\text { Niveau } \\
\text { de signification } \\
\text { Significance }\end{array}$ \\
\hline $\begin{array}{l}\mathrm{N} \text { ingéré }(\mathrm{g}) \ldots \ldots \\
\text { Ingested } N(g)\end{array}$ & $22,2 \pm 3,3$ & $28,9 \pm 0,9$ & $\mathrm{P}<0,01$ \\
\hline $\begin{array}{l}\mathrm{N} \text { fixé }(\mathrm{g}) \ldots \ldots \ldots \\
\text { Fixed } N(g)\end{array}$ & $6,2 \pm 0,8$ & $7,0 \pm 0,7$ & Non significatif \\
\hline $\begin{array}{l}\text { CUPN }(\%) \\
N P U(\%)\end{array}$ & $28,0 \pm 0,8$ & $24,4 \pm 2,7$ & $\mathrm{P}<0,05$ \\
\hline
\end{tabular}

Tableau 5

Poids moyen par groupe de lapins après jeûne.

Poids et rendements moyens des carcasses ( \pm Ecarts-types).

Mean weight of rabbits after fasting.

Mean weight and dressing percentage of carcass ( \pm Standard Deviations).

\begin{tabular}{|c|c|c|}
\hline & $\begin{array}{l}\text { Aliment I } \\
\text { Diet I }\end{array}$ & $\begin{array}{l}\text { Aliment II } \\
\text { Diet II }\end{array}$ \\
\hline $\begin{array}{l}\text { Poids vif après jeûne }(\mathrm{g}) \ldots \ldots \ldots \ldots \ldots \ldots \\
\text { Body weight after fasting }(\mathrm{g})\end{array}$ & $2819 \pm 209$ & $3284 \pm 304$ \\
\hline $\begin{array}{l}\text { Poids de la carcasse }(\mathrm{g}) \ldots \ldots \ldots \ldots \ldots \\
\text { Carcass weight }(g)\end{array}$ & $1835 \pm 153$ & $2027 \pm 188$ \\
\hline $\begin{array}{l}\text { Rendement de la carcasse }(\%) \ldots \ldots \ldots \\
\text { Dressing percentage }(\%)\end{array}$ & $65,1 \pm 2,3$ & $61,8 \pm 2,0$ \\
\hline
\end{tabular}


L'analyse des échantillons de sang récoltés lors de l'abattage n'a pas révélé de différence significative entre les groupes en ce qui concerne les protéines sériques. Par contre le taux d'urée sanguine est significativement différent $(\mathrm{P}<0,01)$. Curieusement, les animaux du groupe I, dont la teneur en protéines de l'aliment était plus faible, atteignent un taux d'urée plus élevé (tabl. 6).

\section{TABLEAU 6}

Taux moyen par groupe de protéines sériques $(\mathrm{g} / 100 \mathrm{ml})$

et d'urée ( $\mathrm{mg} / 100 \mathrm{ml}$ ) ( \pm Ecarts-types).

Mean protein blood level $(\mathrm{g} / 100 \mathrm{ml})$ and urea blood level $(\mathrm{mg} / 100 \mathrm{ml})$ ( \pm Standard Deviations).

\begin{tabular}{l|c|c}
\hline & $\begin{array}{c}\text { Aliment I } \\
\text { Diet I }\end{array}$ & $\begin{array}{c}\text { Aliment II } \\
\text { Diet II }\end{array}$ \\
\hline $\begin{array}{l}\text { Taux de protéines sanguines } \ldots \ldots \ldots \ldots \ldots \ldots \\
\begin{array}{l}\text { Protein blood level } \\
\text { Taux d'urée sanguine .............. }\end{array}\end{array}$ & $6,9 \pm 0,8$ & $7,0 \pm 0,8$ \\
\begin{tabular}{l} 
Urea blood level \\
\hline
\end{tabular}
\end{tabular}

Les animaux qui ont reçu l'aliment II (riche en protéines) ont des muscles sensiblement plus lourds, plus riches en matière sèche et matière grasse, que ceux des lapins du groupe I (tabl, 7). Cependant, chez ces derniers, la matière azotée totale était plus élevée $(87,7$ p. 100 contre 84,9 p. 100$)$.

Chez les femelles, la teneur en lipides du muscle biceps femoris (M2) est plus élevé que chez les mâles. Dans le cas de l'aliment I, le pourcentage moyen de matière grasse était de 5,5 p. 100 chez les mâles et de 7,1 p. 100 chez les femelles. Chez les individus ayant reçu l'aliment II, nous avons enregistré 8,2 p. 100 chez les mâles et 10,6 p. $100 \mathrm{chez}$ les femelles. Par contre, l'effet du sexe est non significatif $(P>0,05)$ en ce qui concerne la matière sèche et la matière azotée totale.

Pour les deux groupes, les teneurs en matière sèche des muscles de l'épaule (M1) et de la cuisse (M2) sont identiques; mais le muscle de la cuisse est plus riche en matière azotée totale que celui de l'épaule.

Les animaux à croissance rapide (groupe II) déposent sensiblement plus de graisse que ceux du groupe I (tabl. 8). Par contre, la taille des adipocytes de la graisse périrénale est la même quel que soit le régime distribué, mais le nombre de cellules graisseuses par gramme de graisse est plus élevé chez les animaux ayant reçu l'aliment I (tabl. 8).

En valeurs absolues, les longueurs moyennes des segments du tube digestif sont significativement plus élevées chez les animaux du groupe II, à l'exception du côlon qui est équivalent dans les deux groupes. Ramenées au $\mathrm{kg}$ de poids vif, les valeurs sont inversées, sauf dans le cas de l'intestin grêle, où la différence n'est pas significative (tabl. 9). 


\section{TABleau 7}

Poids secs et composition chimique des muscles Triceps (M 1) et Coccygeofemoralis + Biceps femoris (M 2) ( $( \pm$ Ecarts-types).

Dry weight and chemical composition of the muscles Triceps (M 1) and Coccygeofemoralis + Biceps femoris $(M 2)$ ( \pm Standard Deviations).

\begin{tabular}{|c|c|c|c|}
\hline & $\begin{array}{l}\text { Muscle } \\
\text { Muscle }\end{array}$ & $\begin{array}{l}\text { Aliment I } \\
\text { Diet I }\end{array}$ & $\begin{array}{l}\text { Aliment II } \\
\text { Diet II }\end{array}$ \\
\hline $\begin{array}{l}\text { Poids } \sec (\mathrm{g})(1) \\
\text { Dry weight }(g)\end{array}$ & $\begin{array}{l}\text { M1 } \\
\text { M2 }\end{array}$ & $\begin{array}{r}2,5 \pm 0,2 \\
10,4 \pm 1,1\end{array}$ & $\begin{array}{r}2,8 \pm 0,3 \\
12,5 \pm 1,7\end{array}$ \\
\hline $\begin{array}{l}\% \text { de matière sèche } \ldots \ldots \ldots \ldots \ldots \\
\% \text { of dry matter }\end{array}$ & $\begin{array}{l}\text { M1 } \\
\text { M2 }\end{array}$ & $\begin{array}{l}24,1 \pm 1,0 \\
24,6 \pm 0,9\end{array}$ & $\begin{array}{l}25,8 \pm 1,1 \\
25,3 \pm 1,0 \\
\end{array}$ \\
\hline $\begin{array}{l}\% \text { de protéines } / \mathrm{MS} \\
\% \text { of protein } / D M\end{array}$ & $\begin{array}{l}\text { M1 } \\
\text { M2 }\end{array}$ & $\begin{array}{l}79,9 \pm 2,7 \\
87,7 \pm 1,8\end{array}$ & $\begin{array}{l}75,4 \pm 3,6 \\
84,9 \pm 3,2\end{array}$ \\
\hline $\begin{array}{l}\% \text { de lipides/MS (2) } \ldots \\
\% \text { of lipid/DM }\end{array}$ & $\begin{array}{l}\text { M1 } \\
\text { M2 }\end{array}$ & $6,3 \pm 1,6$ & $\overline{9,4 \pm 2,5}$ \\
\hline
\end{tabular}

(1) Après lyophilisation - After freeze-drying.

(2) Le poids trop faible du Triceps (M1) a rendu impossible, après l'évaluation du taux protéique, la détermination de la teneur en lipides - The too low weight of tha Triceps (M1) did not allow, after the protein level evaluation, the determination of the lipid content.

\section{Tableau 8}

Poids moyen, par groupe, des graisses de dépôt, valeurs moyennes, par groupe, du nombre et de la taille des adipocytes de $1 \mathrm{~g}$ de graisse périrénale ( \pm Ecarts-types). Analyse de la variance.

Mean weight of the adipose deposits, mean number and size of adipocytes of perirenal fatty tissue ( \pm Standard Deviations). Variance analysis.

\begin{tabular}{|c|c|c|c|}
\hline & $\begin{array}{l}\text { Aliment I } \\
\text { Diet } I\end{array}$ & $\begin{array}{l}\text { Aliment II } \\
\text { Diet II }\end{array}$ & $\begin{array}{c}\text { Niveau } \\
\text { de signification } \\
\text { Significance }\end{array}$ \\
\hline $\begin{array}{l}\text { Poids des graisses de dépôt }(\mathrm{g}) \text {.. } \\
\text { Weight of adipose deposits }(\mathrm{g})\end{array}$ & $56 \pm 12$ & $66 \pm 25$ & $\mathrm{P}<0,05$ \\
\hline $\begin{array}{l}\text { Nombre d'adipocytes }\left(\times 2.10^{3}\right) \ldots \\
\text { Number of adipocytes }\left(\times 2.10^{3}\right)\end{array}$ & $457 \pm 144$ & $627 \pm 334$ & Non significatif \\
\hline $\begin{array}{l}\text { Rayon des adipocytes }(\mu) \ldots \ldots \ldots \\
\text { Adipocyte radius }(\mu)\end{array}$ & $29 \pm 3$ & $27 \pm$ & Non significatif \\
\hline
\end{tabular}




\section{Tableau 9}

Longueurs moyennes, par groupe, des segments du tube digestif (cm) ( \pm Ecarts-types). Analyse de la variance.

Mean lengths of the digestive tract segments (cm) ( \pm Standard Deviations). Variance analysis.

\begin{tabular}{|c|c|c|c|}
\hline & $\begin{array}{l}\text { Aliment I } \\
\text { Diet I }\end{array}$ & $\begin{array}{l}\text { Aliment II } \\
\text { Diet II }\end{array}$ & $\begin{array}{l}\text { Résultats stat. } \\
\text { Stat. results }\end{array}$ \\
\hline Intestin grêle $(\mathrm{cm})$. & $305,1 \pm 23,4$ & $341,8 \pm 31,2$ & $\mathbf{P}<0,01$ \\
\hline $\begin{array}{l}\text { Small intestine }(\mathrm{cm}) \\
(\mathrm{cm} / \mathrm{kg} \text { P.V.) }\end{array}$ & $103,0 \pm 5,4$ & $98,2 \pm 9,3$ & Non significatif \\
\hline Caecum + appendice $(\mathrm{cm})$ & $47,2 \pm 4,2$ & $50,5 \pm 3,8$ & $\mathrm{P}<0,05$ \\
\hline $\begin{array}{r}\text { Caecum }+ \text { appendix }(\mathrm{cm}) \\
(\mathrm{cm} / \mathrm{kg} \text { P.V. }) \ldots\end{array}$ & $16,0 \pm 1,4$ & $14,54 \pm 1,5$ & $P<0,01$ \\
\hline $\begin{array}{r}\text { Colon }(\mathrm{cm}) \ldots \ldots \ldots \\
(\mathrm{cm} / \mathrm{kg} \text { P.V.) }\end{array}$ & $\begin{array}{r}141,3 \pm 14,3 \\
48,0 \pm 6,8\end{array}$ & $\begin{array}{r}140,7 \pm 11,4 \\
40,4 \pm 3,7\end{array}$ & $\begin{array}{c}\text { Non significatif } \\
\mathrm{P}<0,01\end{array}$ \\
\hline
\end{tabular}

Les poids moyens des quelques organes contrôlés (cœur, poumons, reins, foie et tube digestif vide) ont évolué différemment (tabl. 10). Mais si en valeurs absolues, les poids moyens sont légèrement plus élevés chez les animaux du groupe II, seul le foie demeure plus lourd, lorsque les valeurs sont calculées en fonction du poids vif. Cette différence n'est cependant pas significative $(P>0,05)$.

\section{Tableau 10}

Poids moyens, par groupe, des divers organes et du tube digestif ( \pm Ecarts-types). Analyse de la variance.

Mean weight of the various organs and of the digestive tract ( \pm Standard Deviations). Variance analysis.

\begin{tabular}{|c|c|c|c|}
\hline & $\begin{array}{l}\text { Aliment I } \\
\text { Diet I }\end{array}$ & $\begin{array}{l}\text { Aliment II } \\
\text { Diet II }\end{array}$ & $\begin{array}{l}\text { Résultats stat. } \\
\text { Stat. results }\end{array}$ \\
\hline $\begin{array}{r}\text { Cour }-\underset{(g e a r t}{\operatorname{He}}(\mathrm{g}) \\
(\mathrm{kg} \text { P.V.) }\end{array}$ & $\begin{array}{l}6,8 \pm 1,0 \\
2,3 \pm 0,3\end{array}$ & $\begin{array}{l}7,7 \pm 1,0 \\
2,1 \pm 0,2\end{array}$ & $\begin{array}{c}P<0,01 \\
\text { Non significatif }\end{array}$ \\
\hline $\begin{array}{r}\text { Poumons - Lungs (g) } \ldots \ldots \\
(\mathrm{g} / \mathrm{kg} \text { P.V.) } \\
\ldots \ldots \ldots\end{array}$ & $\begin{aligned} 11,7 & \pm 1,9 \\
4,0 & \pm 0,8\end{aligned}$ & $\begin{array}{r}12,0 \pm 2,3 \\
3,4 \pm 0,5\end{array}$ & $\begin{array}{c}\text { Non significatif } \\
\quad P<0,01\end{array}$ \\
\hline $\begin{array}{r}\text { Reins - Kidneys (g) } \ldots \ldots \ldots \\
(\mathrm{g} / \mathrm{kg} \text { P.V. }) \ldots \ldots \ldots\end{array}$ & $\begin{array}{r}14,9 \pm 2,7 \\
5,1 \pm 1,1\end{array}$ & $\begin{array}{r}15,9 \pm 1,6 \\
4,5 \pm 0,3\end{array}$ & $\begin{array}{l}\text { Non significatif } \\
\quad P<0,05\end{array}$ \\
\hline $\begin{aligned} & \text { Foie }-\underset{\text { Liver }}{(\mathrm{g})} \ldots \ldots \ldots \ldots \\
& \quad(\mathrm{g} / \mathrm{kg} \quad \text { P.V. } \ldots \ldots \ldots\end{aligned}$ & $\begin{array}{l}71,7 \pm 11,7 \\
24,4 \pm 4,6\end{array}$ & $\begin{array}{l}92,3 \pm 13,0 \\
26,3 \pm 3,1\end{array}$ & $\begin{array}{c}\mathrm{P}<0,01 \\
\text { Non significatif }\end{array}$ \\
\hline $\begin{array}{l}\text { Tube digestif vide }(\mathrm{g}) \ldots \ldots \\
\text { Empty digestive tract }(\mathrm{g}) \\
\qquad(\mathrm{g} / \mathrm{kg} \text { P.V.) } \ldots \ldots \ldots\end{array}$ & $\begin{array}{r}279,9 \pm 36,6 \\
89,7 \pm 9,8\end{array}$ & $\begin{array}{r}301,6 \pm 49,0 \\
90,6 \pm 9,6\end{array}$ & $\begin{array}{c}\text { P }<0,01 \\
\text { Non significatif }\end{array}$ \\
\hline
\end{tabular}


La composition différente des régimes n'a pas influencé les poids secs des deux os étudiés. Par contre, le développement du squelette fut sensiblement plus accusé chez les animaux du groupe I, si l'on compare les longueurs moyennes des humérus et des fémurs des individus des deux groupes (tabl. 11). De plus, les humérus des lapins du groupe I sont significativement $(\mathrm{P}<0,05)$ plus chargés en calcium, bien que le rapport calcium/phosphore ne soit pas significativement différent.

\section{Tableau 11}

Mesures moyennes de l'humérus gauche et du fémur gauche ( \pm Ecarts-types). Analyse de la variance.

Mean weight and size of the left humerus and the left femur ( \pm Standard Deviations). Variance analysis.

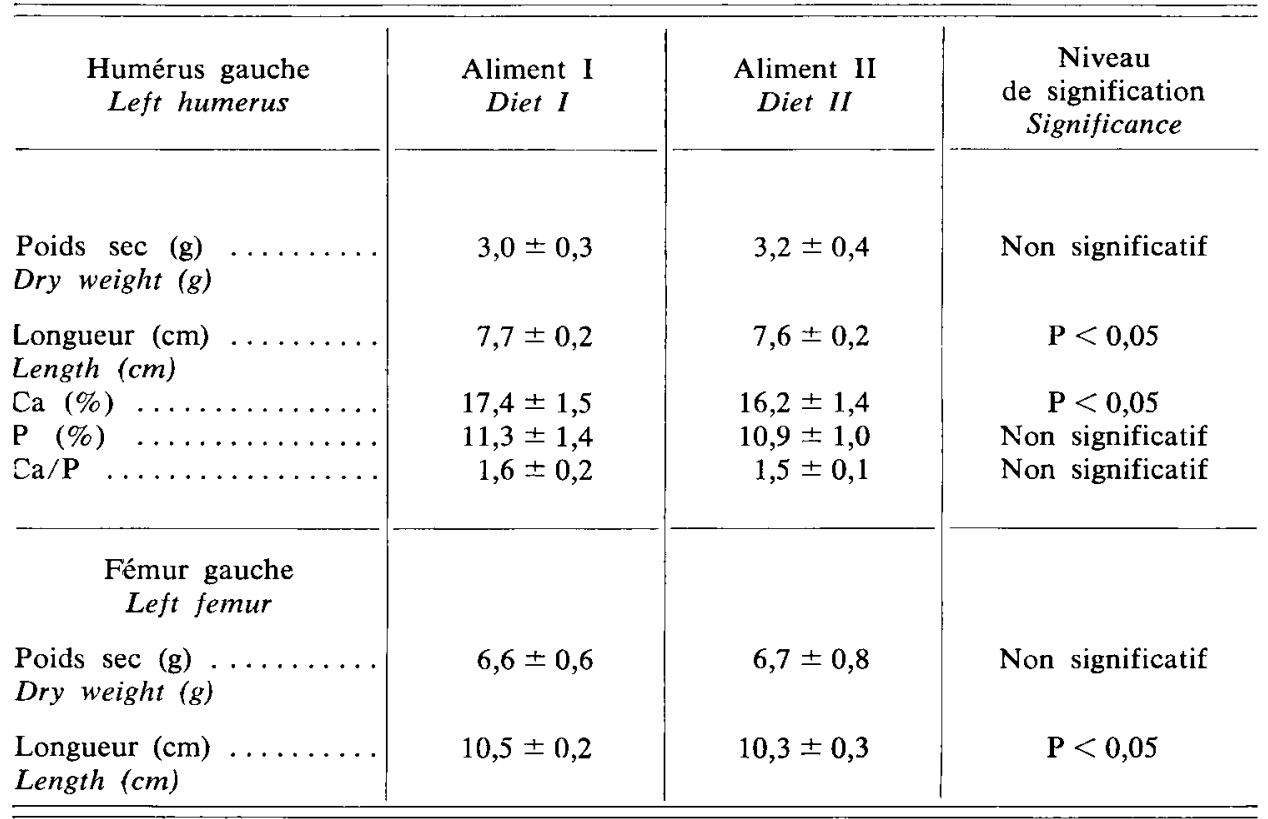

\section{Discussion}

Les conclusions qui peuvent être tirées de ce travail sont dépendantes de la conduite expérimentale.

Les performances des lapins recevant l'aliment II (riche en protéines) ont été nettement supérieures à celles des animaux qui ont reçu l'aliment $I$. Le faible taux de cellulose de l'aliment II (12,4 p. 100), minimum proposé d'ailleurs par Lebas (1979) et Arveux (1980), ne semble pas avoir pénalisé la croissance des animaux du groupe II. Nous n'avons pas davantage observé les troubles digestifs décrits par 
plusieurs auteurs (Heckmann \& Mehner, 1970 ; Renault, 1975 ; Davidson, 1977 ; SPREADbURY \& Davidson, 1978), dans le cas bien précis d'une carence en cellulose dans la ration. Il est fort probable que l'initiation précoce des lapereaux à leur régime expérimental explique en grande partie la réussite de l'expérimentation.

Une forte élévation du taux de protéines $(27,0$ p. 100) associée à la diminution du taux de cellulose $(12,4$ p. 100) a entraîné une amélioration de la croissance et de l'indice de consommation (IC). Cette observation n'est qu'en partie comparable à celle de Lebas (1973 a) qui, en utilisant des rations de 17 et 23 p. 100 de protéines de soja, obtient des résultats orientés dans le même sens que les nôtres, mais avec des différences insuffisantes pour lui permettre de conclure que la croissance et l'IC sont améliorés, lorsque le taux de protéines dépasse 2 p. 100 de la ration. De plus, Lebas (1973 a) a testé des rations isocellulosiques et ses observations ont porté sur des périodes courtes et des animaux jeunes. Par contre, nos conclusions s'accordent mieux avec celles de Heckmann \& Mehner (1970) qui constatent également que l'IC s'améliore lorsque le taux de protéines varie de 18 à 28 p. 100. BOMBEKE et son équipe (1975), observant des lapins de la même race que les nôtres, ont obtenu, avec une ration contenant 10,89 p. 100 de cellulose et 20,57 p. 100 de protéines, un IC de 3,1 pour la période de 3 à 11 semaines. Dans notre cas, avec une augmentation de 7 unités du taux de protéines $(27,0$ p. 100) et un taux légèrement supérieur de cellulose (12,4 p. 100), nous obtenons un IC de 3,22.

Au terme de la période expérimentale, les quantités totales d'aliment consommé par les deux groupes, de la $1^{\text {re }}$ à $1 \mathrm{a} 13^{\circ}$ semaine après le sevrage, sont presqu'égales. Par contre, la répartition des consommations dans le temps a différé selon l'aliment distribué. Cette observation est à rapprocher de l'évolution des gains de poids dans le temps. Jusqu'à la $6^{\text {c }}$ semaine après le sevrage ( 30 jours), les deux groupes présentent des gains de poids, et donc des besoins énergétiques pour couvrir ceux-ci, pratiquement égaux. La courbe de consommation supérieure des lapins du groupe I durant cette période (fig. 3), montre que, des animaux d'une même race, pour assurer une croissance équivalente, ingèrent une plus grande quantité d'aliment pauvre en énergie. Les lapins sont capables d'ajuster leur consommation en fonction de leurs besoins énergétiques, pour autant que le taux d'énergie des aliments soit compris dans certaines limites : $2500-3000 \mathrm{kcal}$ ED/kg (SPrEAdbury \& Davidson, 1978 ; Lebas, 1979 ; Dehalle, 1980, 1981 ; Lebas et al., 1982).

A partir de la $6^{\circ}$ semaine après le sevrage, la croissance des lapins du groupe II se détache nettement, tandis que leur consommation, jusqu'alors inférieure à celle du groupe I, devient supérieure et le reste jusqu'à la fin de l'expérience. Au-delà de la $6^{\circ}$ semaine, il semble que les lapins du groupe II commencent véritablement à tirer profit de la richesse en protéines de l'aliment II et, moyennant une augmentation simultanée de la consommation, maintiennent un rythme de croissance rapide, que les animaux recevant l'aliment I, n'ont pas suivi. Chez ces derniers, il pourrait s'agir d'un effet retardé d'une insatisfaction du besoin azoté chez le jeune animal. L'on pourrait suggérer également une réduction de l'appétence de l'aliment I en raison de l'utilisation de la paille comme composante de substitution. Mais cet aliment n'ayant pas été déprécié durant la première partie de l'expérience, la question se poserait de savoir pourquoi, à un certain âge son appétence diminuerait ? MERcIER et al. (1980) ont par ailleurs démontré que les vitesses de croissance ne sont pas affectées par le taux d'incorporation de la paille, qu'elle soit traitée ou non.

La $6^{\circ}$ semaine après le sevrage semble être une période critique chez le lapin. 
Il est intéressant de noter à ce propos que LeBAs (1973 b) avait observé en comparant deux races de lapins, les races californienne et néo-zélandaise, une diminution appréciable des CUDa, considérés entre la $6^{\prime \prime}$ et la $9^{\prime \prime}$ semaine d'âge.

En considérant l'utilisation digestive des aliments, nous constatons que les MS et MAT de l'aliment II sont significativement mieux digérées que celles de l'aliment I, et que les animaux du groupe II ont fixé, en valeur absolue, plus de protéines que ceux du groupe I. Par contre, chez les lapins du groupe I (aliment pauvre en protéines), nous observons une tendance à mieux digérer la cellulose. Ces résultats sont probablement la résultante de deux facteurs avancés par plusieurs auteurs (Besedina \& Perel'dik, 1970 ; Agullera, 1973 ; Colin, 1974 ; Lebas, 1975 ; Rossilet, 1977 ; Carregal, 1979) : d'une part, l'augmentation du taux de cellulose diminue la digestibilité de la ration, en général, mais favorise sa propre digestibilité, alors que celle de l'azote n'est pas modifiée de manière significative, et, d'autre part, l'élévation du taux de matières azotées améliore la digestibilité apparente de l'azote.

En valeur relative, cependant, les résultats sont inversés. Ce sont, en effet, les animaux qui ont disposé de moins. de protéines (groupe 1), qui les ont utilisées le mieux. Cette plus grande efficacité protéique chez les animaux qui en reçoivent le moins est un phénomène bien connu chez les monogastriques (Colin, 1974 ; Ouhayoun \& Delmas, 1980; Dehalle, 1981; Ouhayoun \& Cheriet, 1983). La cellulose constitue, en effet, un substrat énergétique, qui favorise davantage la synthèse de protéines microbiennes chez des animaux du groupe II, une partie des protéines apparemment absorbées en supplément par rapport aux lapins du groupe I, a pu l'être sous forme ammoniacale.

Nos observations s'accordent également avec celles de Spreadbury (1978), qui constate que le CUPN atteint un maximum à 15 p. 100 de protéines, équilibrées en acides aminés, dans la ration, puis diminue. Au-delà de l'optimum, la quantité d'azote fixé continue à augmenter mais à un rythme beaucoup plus faible.

Les taux de protéines sériques, mesurés à 121 jours, sont semblables pour les deux groupes. Ils sont en agrément avec les données de Lebenhartz (1974), qui avait testé un aliment contenant 15 à 17 p. 100 de protéines. Par ailleurs, les taux d'urée sanguins s'accordent avec les valeurs obtenues par Ross (1949). Au moment de l'abattage, le taux moyen d'urée est sensiblement supérieur chez les animaux recevant l'aliment pauvre en protéines (groupe I), ce qui peut paraître étonnant. Il est probable que, si à 2 mois et demi une utilisation efficace de la ration $I$ est nécessaire pour assurer les $219 \mathrm{~g}$ de gain de poids hebdomadaire (GMH), ce n'est plus le cas à 4 mois, lorsque le GMH du groupe I n'est plus que de $81 \mathrm{~g}$. A ce moment, le surplus d'azote serait éliminé par l'urine. Au contraire, les animaux du groupe II, avec un GMH de $288 \mathrm{~g}$ à 2 mois et demi et de $200 \mathrm{~g}$ à 4 mois, seraient obligés de maintenir un CUPN relativement élevé.

Les animaux qui ont reçu l'aliment riche en protéines ont des muscles légèrement plus lourds, que ceux de l'autre groupe. L'augmentation du taux de matière grasse (de 6,3 à 9,4) est du même ordre de grandeur que la diminution du taux de matière azotée totale. Ce sont également les animaux à croissance plus rapide (groupe II) qui ont déposé le plus de graisse corporelle $(66 \mathrm{~g}$ contre $56 \mathrm{~g}$ ). Ces observations vont dans le même sens que les résultats de Prud'Hon et al. (1970) et de Ouyahoun et al. $(1979,1980)$, quoique ces auteurs aient obtenu de pareilles conclusions à partir de protocoles différents. 
Les mesures des poids et des longueurs montrent que le régime riche en fibres favorise le développement de certains segments du tube digestif. Cela confirme les observations de Hoover \& Heitmann (1972), Arveux (1980) et Rossilet (1977). Ce développement sensible favorisé par un taux de cellulose plus élevé est surtout apparent lorsque l'on considère les valeurs relatives (longueur $/ \mathrm{kg} P V$ ). Tous les organes mesurés (intestin grêle, caecum + appendice, côlon) ont en effet des longueurs moyennes par $\mathrm{kg}$ de poids vifs inférieures chez les lapins du groupe II.

Bien que le poids du tube digestif soit nettement plus lourd en valeur absolue chez les animaux du groupe II, il n'est plus significativement différent en valeur relative, ce qui laisserait supposer que la différence de rendement observée serait due strictement aux contenus résiduels variables chez les animaux à jeûn.

En ce qui concerne le foie, les poids bruts et relatifs sont plus élevés chez les animaux ayant reçu l'aliment II, riche en protéines. Des restrictions alimentaires temporelles entraînent également chez le lapin une hypertrophie hépatique, qui serait la conséquence d'un effort métabolique accru pour préserver son homéostasie (LEBAS et al., 1982). Dans notre cas, le foie des lapins qui ont le plus consommé est plus lourd, et cette légère hypertrophie pourrait être le résultat d'une activité métabolique différente, compensatoire d'une efficacité protidique significativement plus faible $(P<0,05)$ de l'aliment II.

L'apport minéral en calcium dans les aliments dépassait légèrement l'optimum recommandé par BESANçon \& LEBAS (1969). L'aliment I en contenait 1,14 p. 100 et l'aliment II, 1,19 p. 100. L'incorporation supérieure du calcium dans l'humérus chez les individus du groupe I, par rapport à ceux du groupe II, est inattendue et n'est présentement pas explicable, étant donné que les pertes urinaires et fécales n'ont pas été analysées.

Aucun effet du sexe n'a été mis en évidence en ce qui concerne les paramètres étudiés, quel que soit l'aliment distribué. Etant donné que la diversité des phénomènes métaboliques liés à la maturité sexuelle n'apparaît qu'entre 5 et 8 mois, chez le lapin Blanc de Termonde, cette dernière observation n'a rien d'étonnant.

En conclusion, cette expérimentation confirme les très larges possibilités du lapin dans l'ajustement de son ingestion alimentaire, lorsqu'il se trouve en présence de régimes très différents, surchargés ou carencés, en tout ou en partie. Il semble cependant que cette souplesse d'adaptation soit limitée au très jeune âge, et que le lapereau, initié précocement à un régime déséquilibré, augmente ses chances d'adaptation.

Reçu en janvier 1985.

Accepté en février 1986.

\section{Summary}

Effect of a protein rich, low fibre diet on growth performance, organ development and carcass composition of the Termonde White rabbit

The present experiment was carried out to study the adaptation capacity of the rabbit to an unbalanced diet and to determine to what extent dietary components are used by this animal. 
Two groups of 18 Termonde White rabbits of both sexes in equal number were fed ad libitum either an experimental diet (group II) or a control diet (group I) several days before weaning until the age of 120 days. The control diet (diet I) contained 18.9 p. 100 protein and 20.2 p. 100 crude fibre. The experimental diet (diet II), very unbalanced, had a protein excess $(27.0$ p. 100) and a rather low fibre content (12.4 p. 100). Weight gain, feed efficiency, development of some organs, carcass composition and fat deposits were measured.

Rabbits became adapted to both diets as shown by the absence of disease and growth performance. Rabbits fed the protein rich diet II reached a higher mean liveweight $(3491 \mathrm{~g})$ than those fed diet I $(2965 \mathrm{~g})$. Feed conversion ratio of group II was better (3.22) than that of group I (3.78). Apparent digestibility coefficient of dry matter and crude protein was higher with diet II than with diet I. However, rabbits fed the control diet exhibited a higher net protein utilization.

Fat deposits were larger in rabbits fed diet I1. The dressing percentage (warm carcass) was lower in the latter $(61.8 \mathrm{p} .100)$ than in group I $(65.1 \mathrm{p} .100)$. The mean carcass weight of group II was higher and the muscular tissue was richer in dry matter and lipids. By contrast, the protein content of muscular tissue was higher in rabbits fed the control diet (group I).

The sex of the animals did not affect the parameters studied whatever the diet.

Key words : rabbits, protein rich and low fibre diet, growth performance, carcass composition.

\section{Références bibliographiques}

AgUiLeRA J.F., 1973. The influence of the dietary protein level on the digestibility, nutritive value and nitrogen balance in growing rabbits. Congrès International de Cuniculture, Côme (Italie), 364.

Arveux P., 1980. Les besoins en cellulose du lapin. Cuniculture, 31, 13-14.

Besançon P., Lebas F., 1969. Utilisation digestive réelle et rétention du Ca par le lapin en croissance recevant un régime riche en $\mathrm{Ca}$ et $\mathrm{P}$. Ann. Zootech., 18, 437-443.

Besedina G., Perel'dik N.S., 1970. Proportion of the fiber in the diet and digestibility of feeds. Krolik. Zver., 6, 3-5.

Bombeke A., Okerman F., Moermans R.S., 1975. Etude comparative de différentes races de lapins. Revue de l'agriculture, 2, 421-438.

Carregal R.D., 1979. Effects of different fiber contents on digestibility of nutrients by rabbits. Cientifica, 7, 137-142.

Colın M., 1974. Supplémentation en Lysine d'un régime à base de tourteau de sésame chez le lapin. Effets sur les performances de croissance et le bilan azoté estimé par deux méthodes. Ann. Zootech., 23, 119-132.

DAvidson J., 1977. Recent studies in rabbit nutrition. The Rowett Research Institute, Aberdeen (Scotland), 33, 120-125.

de Blas J.C., Torres A., Fraga M.J., Perez E., Galvel J.F., 1977. Influence of weight and age on the body composition of young doe rabbits. J. Anim. Sci., 45, 48-53.

Dehalle C., 1980. Concentration en énergie et protéines des aliments : effets sur les performances de croissance du lapin. Congrès Mondial de Cuniculture, Barcelone, vol. II, 56-64.

Dehalle C., 1981. Equilibre entre les apports azotés et énergétiques dans l'alimentation du lapin en croissance. Ann. Zootech., 30, 197-208.

Heckmann F.W., Mehner A., 1970. Versuche über den Eiweiss- und Rohfasergehalt in Alleinfutter für Fungmatkaninchen. Arch. Geflügelzucht Kleintierk, 19, 29-43.

Hoover W.H., Heitman R.N., 1972. Effects of dietary fiber levels on weight gain, caecal volume and volatile fatty acid production in rabbit. J. Nutr., 102, 375-379.

Jentsch W., Schiemann R., Hoffman L., Nehring K., 1963. Utilization of the energy of concentrates by rabbits. Arch. Tierernähr., 13, 133-145. 
Lebas F., 1973 a. Effets de la teneur en protéines de rations à base de soja ou de sésame sur la croissance du lapin. Ann. Zootech., 22, 83-92.

LEBAS F., 1973 b. Variation chez le lapin des CUDa de la matière sèche, matière organique et des matières azotées en fonction de l'âge, du sexe et de la race. Ann. Biol. Anim. Biochim. Biophys., 13, 767-768 (Abstr.).

Lebas $\mathrm{H}^{i}$, 1975 a. Le lapin de chair : besoins nutritionnels et alimentation pratique. Document ITAVI, Paris, 5-49.

Lebas F., 1975 b. Influence de la teneur en énergie de l'aliment sur les performances de croissance du lapin. Ann. Zootech., 24, 281-288.

Lebas F., 1979. Nutrition et alimentation du lapin : les besoins. Cuniculture, 28 (6-4), 159-160.

Lebas F., Laplace J.F., Droumene P., 1982. Effets de la teneur en énergie de l'aliment chez le lapin. Variations en fonction de l'âge des animaux et de la séquence des régimes alimentaires. Ann. Zootech., 31, 233-256.

LebenharTz Ya. Z., 1974. Some value of protein and fat metabolism in rabbits given different amounts of protein (en russe). Svinovodstvo, 2, 76.

Mercier P., Seroux M., Franck Y., 1980. Utilisation de la paille par le lapin à l'engraissement. $\mathrm{II}^{*}$ Congrès Mondial de Cuniculture, Barcelona, 2, 136-145.

Olivier G., 1960. Pratique anthropologique. Vigot Frères, Paris, VI ${ }^{\circ}, 202-203$ et 236-237.

Ouhayoun J., Delmas D., Lebas F., 1979. Influence du taux protéique de la ration sur la composition corporelle du lapin. Ann. Zootech., 28, 453-458.

Ouhayoun J., Delmas D., 1980. Influence du niveau protéique du régime sur le développement corporel de lapins néozélandais. Congrès Mondial de Cuniculture, Barcelone, vol. II, 37-42.

Ouhayoun J., Cheriet S., 1983. Valorisation comparée d'aliments à niveaux protéiques différents, par des lapins sélectionnés sur la vitesse de croissance et par des lapins venant d'élevages traditionnels. I. Etude des performances de croissance et de la composition du gain de poids. Ann. Zootech., 32, 257-276.

Prud'hon M., Vezinhet A., Cantier J., 1970. Croissance, qualités bouchères et coûts de production des lapins de chair. Bull. Tech. Inf., 248, 1-10.

Renault R., 1975. Pathologie digestive du lapin. Le lapin, règles d'élevage et d'hygiène. Inf. Tech. Serv. Vét., 51, 129-142.

Reyne Y., Salcedo-Miliani V.H., 1981. Le lapin peut-il équilibrer seul son ingestion de cellulose ? Cuniculture, 37, 25-28.

Ross E.J., 1949. Cité dans Biology Data Book, éd. par Altman \& Dittmer, Fed. of American Soc. for Experim. Biol., Bethesda, Maryland, 1974, p. 2029.

Rossilet A., 1977. La cellulose dans l'alimentation du lapin; utilisation digestive et aspects nutritionnels. Thèse de Docteur-Ingénieur, Université Paul et Marie Curie, Paris, 24-49.

Soyeux-Salavize A., 1976. Alimentation du lapin de chair. Les dossiers de l'élevage, tome 1, $1,3-9$.

Spreadbury D., 1978. A study of the protein and amino-acid requirements of the growing New-Zealand White rabbit with emphasis on lysine and sulphur containing amino-acid. Brit. J. Nutr., 39, 601-613.

Spreadbury D., Davidson J., 1978. A study of the need of fibre by the growing NewZealand White rabbit. J. Sci. fd. Agric., 29, 640-648. 